\title{
Survey on Routing Protocols for Under Water Sensor Networks
}

\author{
Kifayat Ullah Jan, Zahoor Jan* \\ Department of Computer Science, Islamia College Univesity Peshawar
}

\begin{abstract}
There are certain routing protocols in underwater sensor network. Providing efficient protocols for underwater sensor networks is a challenging factor. UNSW often uses acoustic channels instead of Radio channels. This paper surveys some routing protocols, and presents a classification of the various approaches which follow among that location based is the main concept.

Keywords: - UNSW, Terrestrial, Acoustic channels.
\end{abstract}

\section{Introduction:-}

Sensor networks used for underwater communication are different in many aspects from traditional wired or even terrestrial sensor networks (Akyildiz et al., 2005 [4]; Heidemann et al., 2006 [5]). Firstly, energy consumptions are different because some important applications require large amounts of data, but very infrequently. Secondly, these networks usually work on a common task instead of representing independent users. The ultimate goal is to maximize the throughput rather than fairness among the nodes. Thirdly, for these networks, there is an important relationship between the link distance, number of hops and reliability. For energy concerns, packets over multiple short hops are preferred instead of long links, as multi-hop data deliveries have been proven more energy efficient for underwater networks than the single hop (liang, 2008 [6]). Underwater Wireless Sensor Networks (UWSN) and terrestrial Wireless Sensor Networks (WSN) share common properties but they have many differences too. These differences necessitate specialized new protocols for underwater communication. The cost of the nodes is one of the differences, as underwater wireless sensors are expensive partially of their more complex transceivers. Another difference relates to the deployment costs. Deployment of underwater sensors, especially in deep waters, can be difficult and expensive. Therefore, UWSN has to be carefully studied and planed (in terms of performance evaluations, simulations, and tests) before its deployment. Power is another important difference since underwater communications require higher power than the terrestrial network. But, the most important difference is the communication medium. Underwater communications cannot use Radio Frequency (RF) signals since they have an enormous attenuation in the Subaquatic medium. Therefore, acoustic signals are used underwater. Routing in WSNs is very challenging due to the inherent characteristics that distinguish these networks of other wireless networks like mobile ad hoc networks or cellular networks. First, due to the relatively large number of sensor nodes, it is not possible to build a global addressing scheme for the deployment of a large number of sensor nodes as the overhead of ID maintenance is high. Thus, traditional IP-based protocols may not be applied to WSNs. Furthermore, sensor nodes that are deployed in an ad-hoc manner need to be self-organizing as the ad-hoc deployment of these nodes requires the system to form connections and cope with the resultant nodal distribution especially that the operation of the sensor networks is unattended. [4] Many new algorithms have been proposed for the routing problem in WSNs. These routing mechanisms have taken into consideration the inherent features of WSNs along with the application and architecture requirements.

\section{Routing Protocols for Under Water Sensor Networks:}

Major Routing Protocols Proposed for Underwater acoustic Sensor Networks are below:-

- Vector Based Forwarding (VBF): - In VBF [1], for handling the problems of packet losses and node failures the data packets are forwarded along redundant and interleaved paths from the source to the destination. It is assumed that every node already knows its location and each packet carries the location of all nodes involved. The forwarding path is virtually a routing pipe and the nodes inside this pipe are eligible for packet forwarding. 


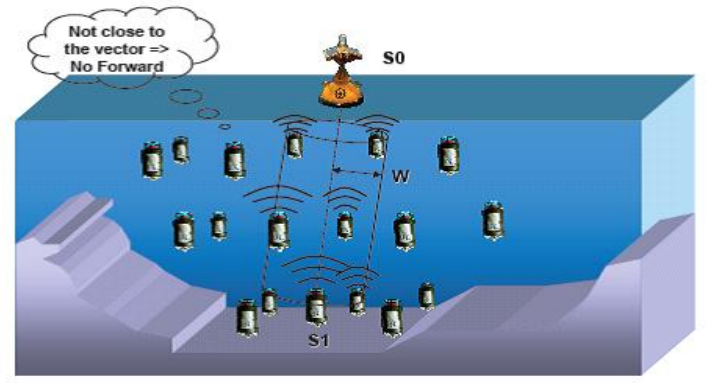

In VBF state information at each nodes are not required, that's the way to the size of the network they are scalable. By saving the energy in VBF only that nodes along the Forwarding path are involved in packet routing.

- Hop by Hop vector Based Forwarding (HH-VBF):- In this virtual concept is used [2]. Each forwarder is defined by per hop virtual pipe. Based on its current location, every intermediate node makes decisions about the pipe direction. In HH-VBF the routing vector from each sender towards the destination is computed. Upon the receipt of a packet, a node computes a destination. Then the node calculates the distance between the computed vector and itself. In case, the distance between the vector and the node is smaller than the radius of virtual routing pipe, this node becomes eligible for forwarding the candidate forwarder.

- Focused beam routing (FBR):- This protocol [3] for acoustic sensor networks are intended to avoid unnecessary flooding of broadcast queries. In FBR, every node in the network is expected to be aware of its location and every source node is aware of its destination. FBR protocol is a scalable routing technique for multi-hop ad-hoc networks based on location information [Jornet, Stojanovic, and Zorzi, 2008[9]]. It is suitable for networks containing both static and mobile nodes, which are not necessarily synchronized to a global clock. A source node must be aware of its own location and the location of its final destination, but not those of other nodes.

- Reliable and energy balanced routing algorithm (REBAR):- It is a location based protocol [8]. Geographic information is used by the nodes between the sources and sinks to transfer the data. Each node is assigned a unique ID and fixed range REBAR is based on the following assumptions.

- Every node knows its location and of the destination through multi-hop routing.

- $\quad$ Sensed data $i$ are sent to the destination at a specific rate.

- Sector -based routing with destination location prediction (SBR-DLP):- For avoiding flooding forwarding of data packets are done in a hop by hop manner. SBR-DLP tries to achieve destination mobility by assuming that all pre planned movements are known to all nodes before the deployment. The sender decides which will be its next hop using information from the candidate nodes trying to eliminate the problem of having multiple nodes acting as relay nodes. It makes no assumption about the location of the destination node being fixed and accurately known to the sender node. It takes into consideration the entire communication circle to locate the candidate relay node. It does not need to rebroadcast RTS every time it cannot find a candidate node within its transmitting cone. Each node is only aware of its own position and the destination of node's pre-planned movements.

- Direction flooding based Routing (DFR):- This Protocol enhances reliability by packet flooding technique. The assumption is that all nodes know about its own location, location of one hop neighbors and that of the final destination. This protocol enhances reliability by packet flooding technique[12]

- Location aware source Routing (LASR): - In this protocol two techniques are adopted for handling high latency of acoustic channels [13], namely link quality metric and location awareness. All the network information including routes and topology information are passed on in the protocol header. Resultantly header size increases as the hop count between source and sink increases. 


\section{Comparison of routing protocols in UASN}

\begin{tabular}{|l|l|l|}
\hline Routing Protocols & Category & Application \\
\hline VBF & & \\
HH-VBF & Location Based & Energy-efficient \\
FBR & Routing & UASN \\
REBAR & & \\
SBR-DLP & & \\
DFR & & \\
LASR & & \\
\hline
\end{tabular}

\section{Conclusion:-}

In this paper an overall view of the UWSN and different routing protocols used depending on the requirements. After presenting the overview of each routing protocol, available comparisons among them are presented. The comparison is necessary in order to point out which routing protocol is best according to the desirable use. As a general conclusion it was noticed that all of them are energy efficient, they can handle dynamic networks.

\section{References:-}

[1]. Xie P, Cui J-H, Lao L. VBF: vector-based forwarding protocol for Underwater sensor networks. Networking 2006. Networking Technologies, services, and protocols.

[2]. Performance of computer and communication networks; mobile and Wireless communications systems. Berlin/Heidelberg: Springer; 2006a. P. 1216-1221

[3]. Nikolaou N, et al. Improving the robustness of location-based routing for underwater sensor networks. In: Proceedings of the OCEANS. - Europe; 2007.

[4]. I. Akyildiz, W. Su, Y. Sankarasubramaniam, and E. Cayirci, "A survey on sensor networks," IEEE Communications Magazine, Volume: 40 Issue: 8, Pp. 102-114, August 2002.

[5]. J. Heidemann, W. Ye, J. Wills, A. Syed, Y. Li, Research challenges and applications for underwater sensor networking, in: Proceedings of the IEEE Wireless Communications and Networking Conference (WCNC2006), Las Vegas, Nevada, USA, April 3-6, 2006.

[6]. Cheng, X., Shu, H., Liang, Q., and Du, D. H.-C. (2008). "Silent positioning in underwater acoustic sensor networks". IEEE Transactions on Vehicular Technology,Vol. 5, No. 3, pp. 1756-1766.

[7]. Chirdchoo, N., Soh, S. W., and Chua, C. K. (2009). 'Sector-based routing with destination location prediction for underwater mobile networks', in Waina, 2009, International Conference on Advanced Information Networking and Applications Workshops, pp. 1148-1153.

[8]. Jinming C, Xiaobing W, Guihai C. REBAR: a reliable and energy balanced routing algorithm for UWSNs. In: Proceedings of the seventh international conference on grid and cooperative computing, GCC ' $08 ; 2008$.

[9]. Jornet JM, Stojanovic M, Zorzi M. Focused beam routing protocol for underwater acoustic networks. In: Proceedings of the third ACM international workshop on Underwater Networks. San Francisco (California, USA): ACM; 2008.

[10]. Jornet, M. J., Stojanovic, M., and Zorzi, M. (2008). 'Focused beam routing protocol for underwater acoustic networks', in Proceedings of the third ACM International workshop on Underwater Networks, International Conference on Mobile Computing and Networking,pp. 75-82.

[11]. Chris G. \& Economides, A. A. (2011). Comparison of Routing Protocols for Underwater Sensor Networks: A Survey. International Journal of Communication Networks and Distributed Systems, Vol. 7, Issue. 3/4, Inderscience Publishers,Geneva, SWITZERLAND.

[12]. Chirdchoo, N, Wee-Seng S, Kee Chaing C. Sector-based routing with destination location prediction for underwater mobile networks. In: Proceedings of the international conference on advanced information networking and applications workshops, WAINA '09; 2009

[13]. Daeyoup H, Dongkyun K. DFR: directional flooding-based routing protocol for underwater sensor networks. In: Proceedings of the OCEANS; 2008.I. Akyildiz, W. Su, Y. Sankarasubramaniam, and E. Cayirci, "A survey on sensor networks," IEEE 